\title{
Application of hot-stage microscopy Direct Analysis in Real-Time mass spectrometry (HDM) to the analysis of polymers
}

\author{
Gage P. Ashton, Lindsay P. Harding*, Gareth M. B. Parkes*, Sophie E. Pownall \\ Thermal Methods Research Unit, School of Applied Sciences, University of Huddersfield, \\ Queensgate, Huddersfield, UK.
}

Rationale. Polymers are ubiquitous and characterisation of their chemical, thermal and mechanical properties is important in many applications. HDM is a new technique which combines optical measurements with the benefits of ambient ionisation mass spectrometry. Physical and chemical information can be obtained as a function of sample temperature, in real-time.

Methods. Samples were placed on a miniaturised hot-stage between a custom-made DART source and the ion trap mass spectrometer inlet, and subjected to both linear and cycled temperature programmes. Optical images were collected using a digital microscope and mass spectra (positive and negative ion) were recorded simultaneously.

Results. Mass spectra and optical images were used to monitor the thermal expansion and release of volatile oligomers from both medical and domestic grades of silicone. Series of ions separated by $74 \mathrm{~m} / \mathrm{z}$ were observed, consistent with the $\mathrm{SiOMe} 2$ monomer; the median mass of these increased with increasing temperature up to the decomposition point (340 $400 \stackrel{\circ}{\circ}$ ). The abundance of volatile material produced decreased with repeated thermal cycling. The coefficients of thermal expansion were calculated from optical data and were in agreement with conventional measurements $\left(2.7-3.6 \times 10^{-4}{ }^{\circ} \mathrm{C}^{-1}\right)$. Two samples of beach sand analysed for the presence of microplastics were found to contain polyethylene and polystyrene, respectively.

Conclusions. Results indicate that the novel technique of HDM can be successfully applied to the characterisation of a wide range of polymers including those in complex matrices.

\section{Keywords:}

Thermomicroscopy, DART-MS, microplastics, silicone, ambient ionisation 


\section{INTRODUCTION}

Direct Analysis in Real-Time (DART), developed by Cody et al. in 2005, is one of a family of ambient ionisation techniques and is invariably used in conjunction with mass spectrometry to give the combined technique DART-MS. ${ }^{1}$ Conventional DART ionisation uses a heated stream of helium containing metastable ions produced by a corona-to-glow plasma. The gas flow is directed at a sample surface, placed within the source in open air; analyte ions are formed in a chemical ionisation-like process and desorbed whereupon they are drawn into the mass spectrometer inlet. ${ }^{2}$ Various sample introduction methods are available, including metal meshes, Diplt tips ${ }^{\mathrm{TM}}$ and tweezer attachments, amongst others. The benefits of the technique are that minimal sample preparation is required and it is amenable to a wide variety of sample types such as solids, liquids and solutions. The temperature of the helium is userdefinable to optimise analyses for various samples.

Application areas range from forensics ${ }^{3}$ and food ${ }^{4}$ to quality control ${ }^{5}$ and hundreds of papers have been published using DART-MS. A good overview of the fundamentals and broad scope of the technique has been given by Gross. ${ }^{6}$

We have reported previously that the measured temperature of the sample reaches only a fraction of the set temperature of the gas ${ }^{7}$ and to address this problem we have developed a novel combined hot-stage microscopy-DART-MS system (HDM) which uses a miniaturised ceramic hot-stage in the ion source to give much more precise temperature control of samples than that obtained with the DART alone, as well as allowing visualisation of melting and desorption behaviour through the integrated microscope. ${ }^{8}$

Hot-stage microscopy (also called thermomicroscopy) is a well-established thermal analysis technique for studying the optical properties of a material as a function of temperature. ${ }^{9}$ Thermal processes including glass transitions, phase changes, melting and decomposition can be monitored..$^{10}$ Most systems allow for the recording of images either as videos ${ }^{11}$ or a series of pictures taken at set time or temperature intervals. Additional quantitative information can be obtained from changes in colour or reflectance undergone by the sample. ${ }^{12}$

The HDM has been described in detail elsewhere ${ }^{8}$ but comprises three main components (Fig. 1). 


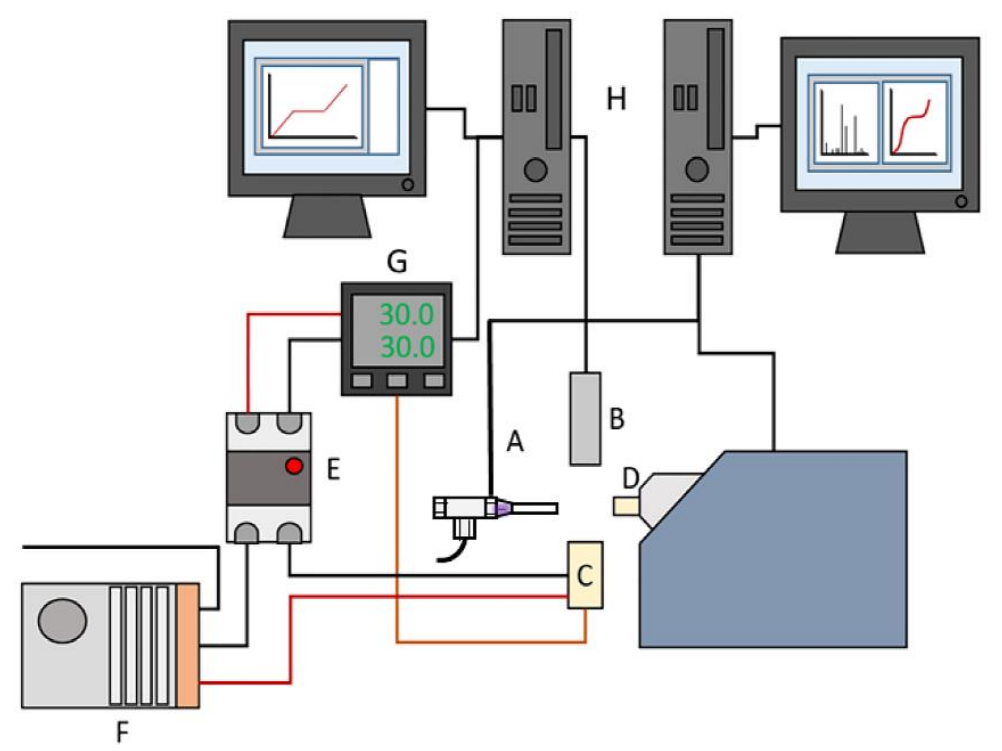

Figure 1 Schematic of HDM system (A: $\mu$-DART source, B: digital microscope, C: hot-stage, D: mass spectrometer, E: solid state relay, F: power supply, G: temperature programmer and H: computers). A photograph of the system is shown in Fig. $\mathbf{S 1 .}$

Since the helium is not required for heating the sample, we have developed a low-flow rate (ca. $0.8 \mathrm{Lmin}^{-1}$ ) ionisation source which we term the $\mu$-DART (Fig. S2). This omits the ion filtering step found in commercially available DART sources. The stream from the source passes over a miniaturised hot-stage constructed from ceramic material and containing a nichrome heating element and low-mass thermocouple. The stage also supports liquid nitrogen cooling and has an overall temperature range of -120 to $700^{\circ} \mathrm{C}$. The final component is a digital pen microscope.

The HDM is controlled by bespoke software developed by the project team. Typically, samples are held in an aluminium pan ( $6 \mathrm{~mm}$ diameter, $3 \mathrm{~mm}$ deep) which is placed upon the hot-stage. In operation the hot-stage follows a selectable temperature programme (which may include heating, cooling and isothermal steps). Images from the microscope are collected at time or temperature intervals as required and recorded as .jpeg files. Real-time colour measurements of selected regions of the images, based on RGB values (red, green and blue pixel values), are obtained. All data are saved in .csv files to allow subsequent processing in Excel. The HDM is not instrument-specific; we are currently using it on Bruker Esquire HCT ion trap and Bruker MicrOToF-q mass spectrometers. The controlled heating and cooling and optical imaging capabilities of the HDM differentiate the system from commercially-available thermal desorption units. ${ }^{13}$ 
Polymers such as silicone, polyethylene, polystyrene etc. are ubiquitous and are found in many applications both domestic and industrial (Fig. 2). As many polymers are poorly biodegradable they tend to persist for many years if they find their way into the environment. ${ }^{14}$ Many tons of plastic enter the sea each year ${ }^{15}$ where they are broken down by the action of weathering to form microplastics, which are defined as particles with diameter between $1 \mathrm{~nm}$ and $5 \mathrm{~mm} .{ }^{16}$ There is substantial concern about the impact that microplastics may have should they enter the foodchain. ${ }^{17}$
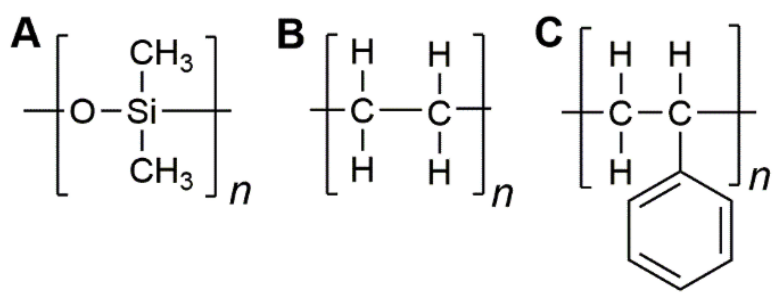

Figure 2: Monomer units of A: silicone; B: polyethylene and C: polystyrene

Currently, pyrolysis GC-MS is a commonly-used method for characterisation of microplastics. ${ }^{18}$ More recently, Majewsky et al. have applied thermal analysis techniques in the detection of polyethylene and polypropylene. ${ }^{19}$ However, there is no universally-accepted analytical procedure for characterisation of these samples.

We present experimental results which demonstrate how the HDM can be used in the analysis of materials, which we exemplify using three discrete polymeric systems. Experiments were carried out on two silicone samples, one used in medical applications and one representative of commonly available cookware. In addition, we show the use of HDM in the analysis of more complex, environmental matrices incorporating multiple polymers.

\section{EXPERIMENTAL}

\subsection{Materials}

\subsubsection{Calibration standards}

Indium, tin and lead metal standards were obtained from LGC (LGC2601, LGC2609 and LGC2608) with certified melting points of $156.6^{\circ} \mathrm{C}, 231.9^{\circ} \mathrm{C}$ and $327.5^{\circ} \mathrm{C}$ respectively.

TuneMix was obtained from Agilent, Cheadle, UK.

\subsubsection{Silicone samples}

Medical grade silicone was obtained from Silex Silicones Ltd, Bordon, UK. 
Domestic silicone bakeware was obtained from Kitchen Corner, Birmingham, UK.

\subsubsection{Microplastics}

Sand samples (ca. $500 \mathrm{~g}$ ) were obtained from the surface of the mid-tidal ranges of two beaches (Scarborough, 54.2828983, -0.3941268 and Reighton, 54.1697530, -0.2485975). Samples were processed using the previously published method of Lindeque et al. utilising density separation to isolate the low-density fractions of the samples. ${ }^{20}$ These were then collected, sieved and washed prior to analysis. Typically, this method recovered between 5 and $25 \mathrm{mg}$ of material per $50 \mathrm{~g}$ of sand, including some sand/shell particulates $(0.05-0.1 \%$ w/w flotation products).

\subsection{Instrument parameters}

The HDM parameters are given for each experiment in the Results and Discussion section. Samples were analysed on a Bruker Esquire HCT ion trap mass spectrometer. The mass range used was $100-2800 \mathrm{~m} / \mathrm{z}$, in alternating polarity mode, using a maximum accumulation time of $200 \mathrm{~ms}$ and a Smart Target of 200,000 . The capillary voltage was set to $4 \mathrm{kV}$ and the source temperature set to $300{ }^{\circ} \mathrm{C}$.

\subsection{Procedure}

\subsubsection{Temperature calibration}

The hot-stage was calibrated using the melting of common thermal analysis calibration standards such as indium, tin or lead. Typically, a sample of the metal $(5-50 \mathrm{mg})$ is heated in a linear fashion and the melting point identified through reflectance changes (measured as changes in RGB values, $\triangle R G B)$.

\subsubsection{Mass spec calibration}

The mass spectrometers were calibrated according to the manufacturer's instructions. Mass and detector calibrations were carried out over a $\mathrm{m} / \mathrm{z}$ range of 100-2700 using TuneMix solution (diluted 1: $10 \mathrm{v} / \mathrm{v}$ in methanol). 


\subsubsection{Silicone analysis}

Medical grade silicone samples were prepared using a metal punching tool to cut thin disks from the bulk material $(\mathrm{h}=0.3 \mathrm{~mm}, \varnothing=4.1 \mathrm{~mm}, 2.6 \mathrm{mg})$.

Domestic bakeware silicone samples were also prepared using the same method ( $h=0.8$ $\mathrm{mm}, \varnothing=4.1 \mathrm{~mm}, 7.6 \mathrm{mg}$ ).

Individual silicone samples were placed within $6 \mathrm{~mm}$ Inconel pans (TA Instruments) and were analysed using the following temperature programmes:

Linear heating experiments were performed at a rate of $10^{\circ} \mathrm{Cmin}^{-1}$ between 30 and $550^{\circ} \mathrm{C}$.

Thermal cycling experiments followed a programme of 50 to $300^{\circ} \mathrm{C}$, held isothermally at 300 ${ }^{\circ} \mathrm{C}$ for 5 minutes then returning to $50^{\circ} \mathrm{C}$ at a heating and cooling rate of $10^{\circ} \mathrm{Cmin}^{-1}$, this cycle was repeated two subsequent times so that the same sample had been exposed to three identical temperature cycles.

\subsubsection{Microplastics analysis}

The collected sand samples were dried in an oven at $70^{\circ} \mathrm{C}$ overnight. The sand was passed through a sieve (mesh $5 \mathrm{~mm}^{2}$ ) to remove large debris and ensure that all polymer particulates collected could be identified as 'microplastics'.

To separate potential microplastics from the bulk of the sand a density separation based on a method reported by Lindeque et al. was performed. ${ }^{20}$ To $50 \mathrm{~g}$ of dried sand was added 150 $\mathrm{mL}$ of a saturated zinc chloride solution $\left(\rho=1.5 \mathrm{gcm}^{-3}\right)$. The solution was then stirred gently for approximately 30 minutes and was then left overnight to allow lower density particulates to float to the surface. The flotation products were then collected on filter paper (Fisherbrand QL100) and washed several times with hot de-ionised water to remove residual zinc chloride. The residue was then dried in an oven at $70^{\circ} \mathrm{C}$ for approximately 30 minutes. The commonlyused oxidative step was not carried out to minimise sample preparation.

Samples of the flotation products (ca. $20 \mathrm{mg}$ ) were introduced to the hot-stage in $6 \mathrm{~mm}$ shallow alumina crucibles (Mettler-Toledo) The samples were exposed to a linear heating experiment in the range of 30 to $550^{\circ} \mathrm{C}$ at a rate of $20^{\circ} \mathrm{Cmin}^{-1}$. 


\section{RESULTS AND DISCUSSION}

\subsection{Medical grade silicone}
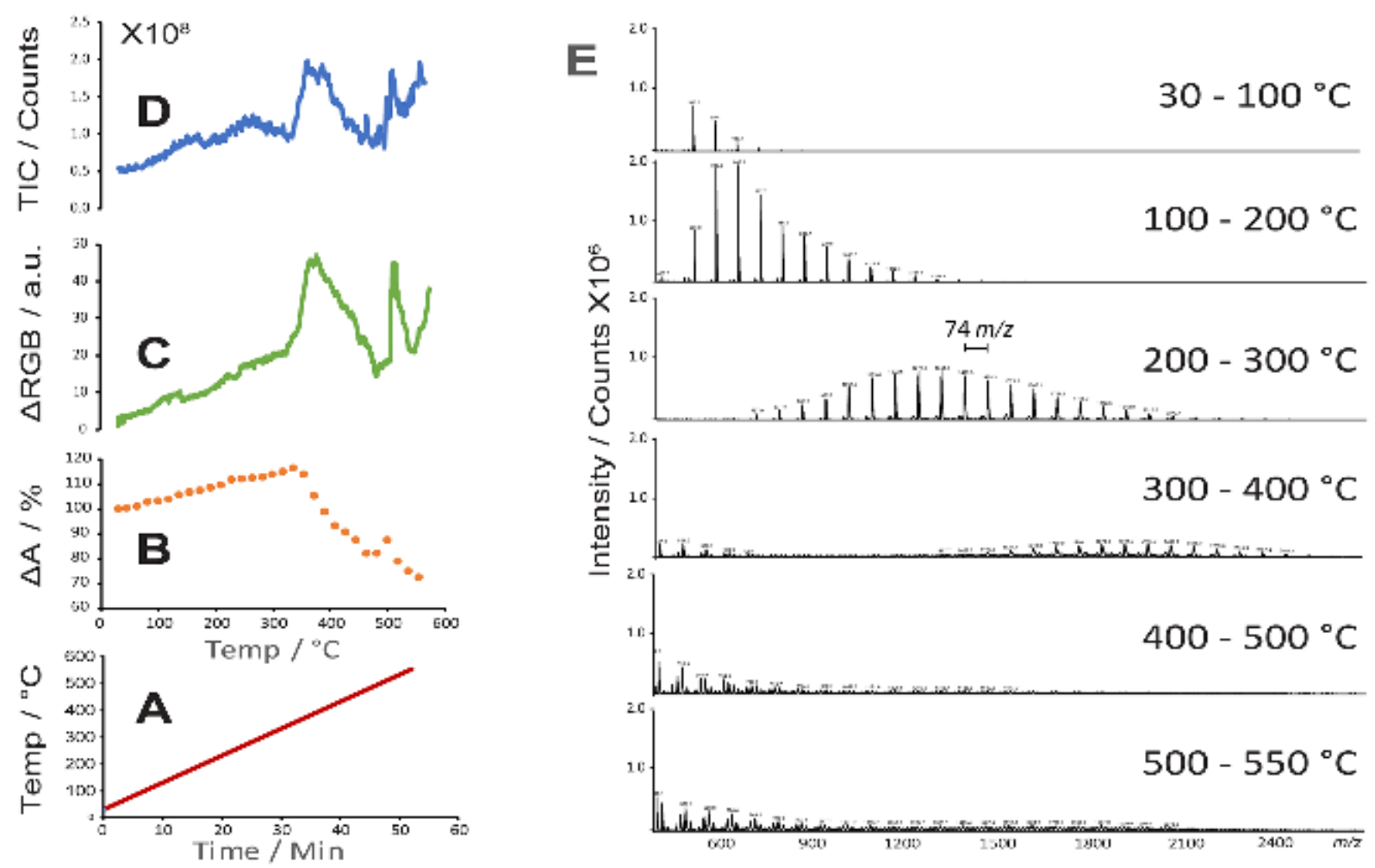

Figure 3 Heating of pharmaceutical grade silicone ( $A$ - temperature, $B-$ area, $C-\triangle R G B$ and $D-T I C, E$ - average mass spectra) (Micrographs in Fig. S3)

Medical grade silicone was subjected to a linear heating programme at $10{ }^{\circ} \mathrm{Cmin}-1$ from 30 to $550 \stackrel{\circ}{\circ}$ (Fig. 3A). Figure 3B shows changes in the relative area of the sample with temperature. It can be seen that the sample expands in a linear fashion until approximately $320{ }^{\circ} \mathrm{C}$ when it shrinks as the sample decomposes (lit. value $339{ }^{\circ} \mathrm{C}$ ).${ }^{21}$ The small event starting at $450{ }^{\circ} \mathrm{C}$ corresponds to the product (silica) cracking, as shown in the micrographs (Fig. S3). These events are mirrored by optical changes as shown in Fig. 3C. Mass spectra were recorded in both positive and negative ion modes across the heating programme; the total ion current (TIC) for the positive ion spectra is displayed in Fig. 3D. Initially there is a steady increase in the number of ions produced up to $320{ }^{\circ} \mathrm{C}$. The TIC increases sharply through the degradation process; the cracking event also releases more trapped, volatile materials. Individual mass spectra (averaged over temperature ranges as indicated) are shown in Fig. 3E. Signals attributable to silicone oligomers are observed, separated by 74 mass units; this represents differences of $\mathrm{SiOMe}_{2}$ monomers (Fig. 2A). It can be seen that the median mass of evolved species increases with increasing temperature until 
decomposition occurs; these results are consistent with previously published studies. ${ }^{22}$ Above $300 \stackrel{\circ}{\circ}$ the first indications of the presence of lower mass decomposition products begin to appear; these persist until the end of the heating process at $550 \stackrel{\circ}{\circ}$.

An understanding of how the dimensions of a sample change with temperature is an important material property. ${ }^{23}$ Data from the linear expansion range (Fig. $3 \mathrm{~B}$ ) were used to calculate the coefficient of thermal expansion (CTE) of the silicone giving a value of $3.6 \times 10^{-}$ ${ }^{4}{ }^{\circ} \mathrm{C}^{-1}$, which is in excellent agreement with the value supplied by the manufacturer $(2-4 \mathrm{x}$ $\left.10^{-4} \stackrel{\circ}{ } \mathrm{C}^{-1}\right) \cdot{ }^{24}$

To gain further insight into the relationship between expansion and oligomer evolution a modified experiment with cyclic heating and cooling between 50 and $300{ }^{\circ} \mathrm{C}$ was performed; the results are shown in Fig. 4. The temperature profile for the experiment is shown in Fig. $4 \mathrm{~A}$ with the corresponding area, optical measurements and TIC shown in Figs $4 \mathrm{~B}, \mathrm{C}$ and $\mathrm{D}$ respectively. Figure $4 \mathrm{E}$ shows the averaged mass spectra over each thermal cycle.
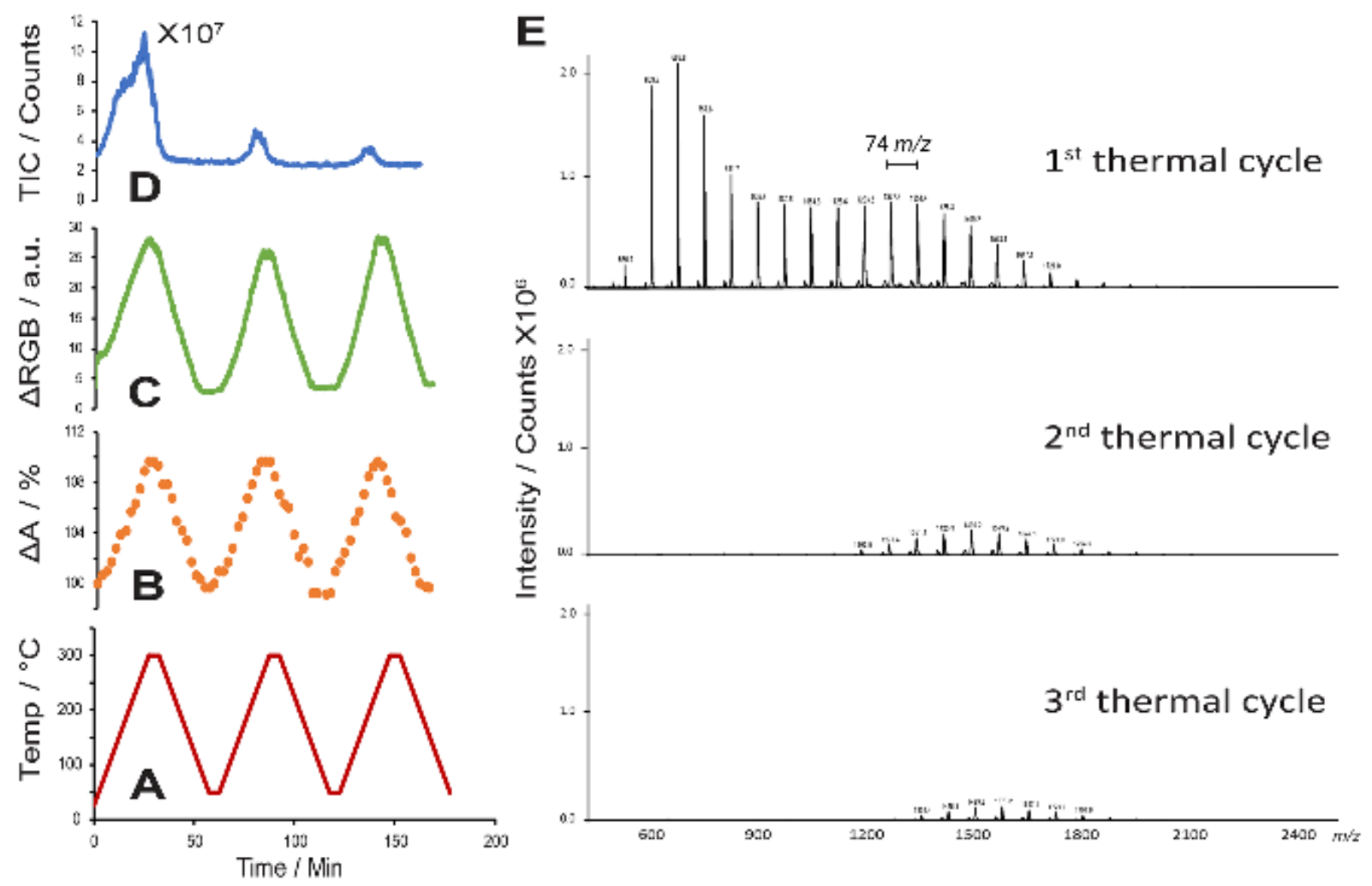

Figure 4 Thermal cycling of pharmaceutical grade silicone ( $A-$ temperature, $B-$ area, $C-\triangle R G B$ and $D$ - TIC, E- average mass spectra) (Micrographs in Fig. S4) 
It can be seen that the expansion and optical changes are completely reversible over three cycles; however, the quantity of volatile material evolved drops markedly after the first cycle and the median mass moves to a higher range (Fig. 4E). This indicates that only looselybound surface species are released over this temperature range as no degradation products are observed.

\subsection{Cookware grade silicone}

Following on from the experiments carried out using medical grade silicone a sample of cookware-grade silicone, designed to be exposed to high temperatures, was examined using identical experiments. Figure 5 shows the results from a linear heating programme from 30 to $550 \stackrel{\circ}{\mathrm{C}}$.
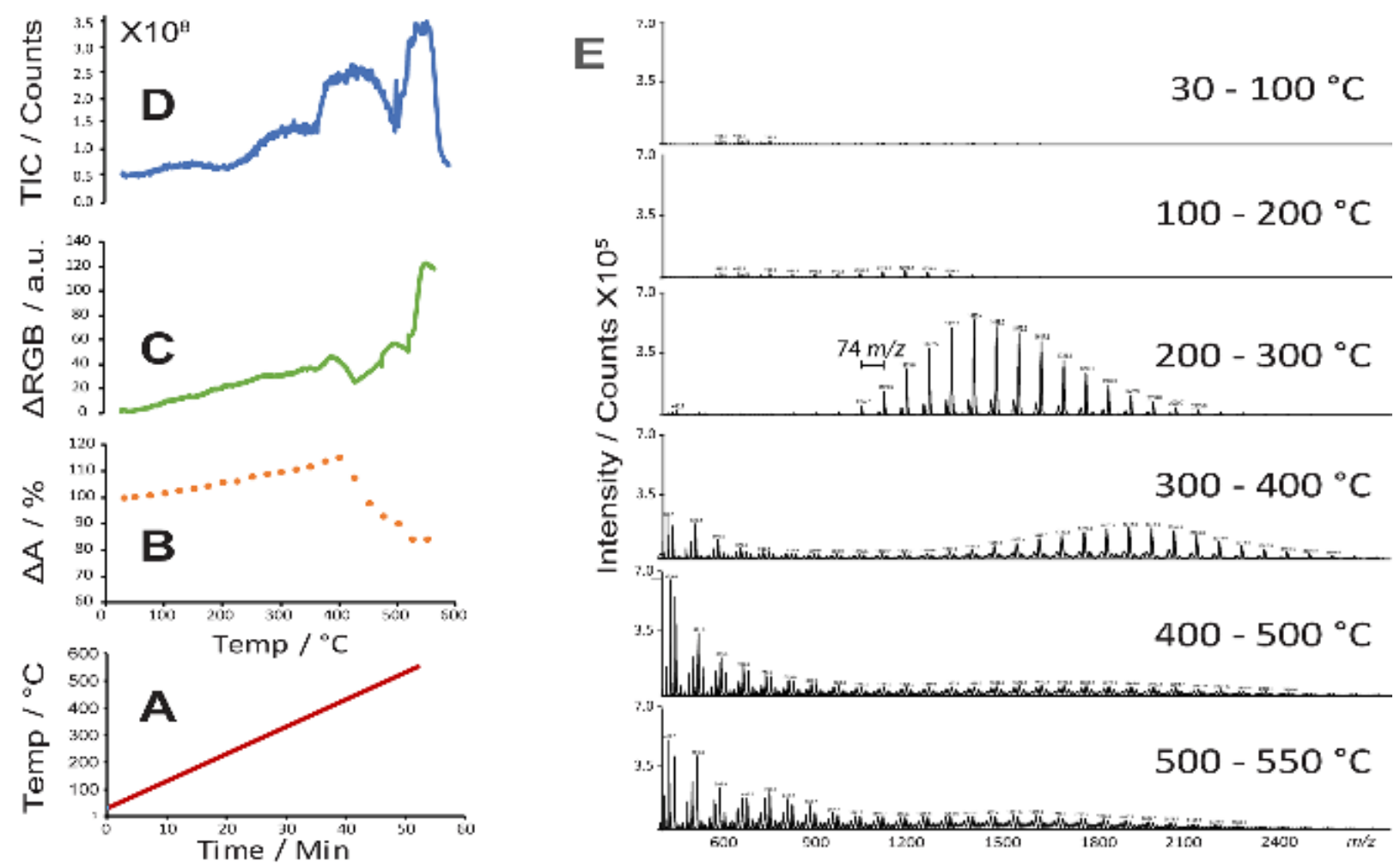

Figure 5 Heating of cookware grade silicone (A - temperature, B-area, C- $\triangle R G B$ and D-TIC, Eaverage mass spectra) (Micrographs in Fig. S5)

The profiles and mass spectra broadly follow those obtained with the medical sample. The same linear expansion is observed, but it can be seen that degradation does not commence until approximately $375^{\circ} \mathrm{C}$. In addition, the cracking process is more pronounced, possibly due to the increased relative thickness of the sample. 
The CTE was again calculated and the value obtained was $2.77 \times 10^{-4}{ }^{\circ} \mathrm{C}^{-1}$. No manufacturer data were available so a sample was analysed using the conventional method of dynamic mechanical analysis; this gave a CTE of $2.73 \times 10^{-4}{ }^{\circ} \mathrm{C}^{-1} .{ }^{25}$

The thermal cycling experiment was repeated and the results are shown in Fig. 6. Again, there are strong similarities to the results gained for the medical sample. It would appear that the measured expansion declines slightly from the first to third cycles, which is a reproducible effect. There is an apparent shoulder on the first cycle in the TIC which is not observed with the medical sample. The corresponding mass spectrum shows a series of low abundance ions consistent with the presence of PEG, which is used as a releasing agent during manufacture. ${ }^{26}$
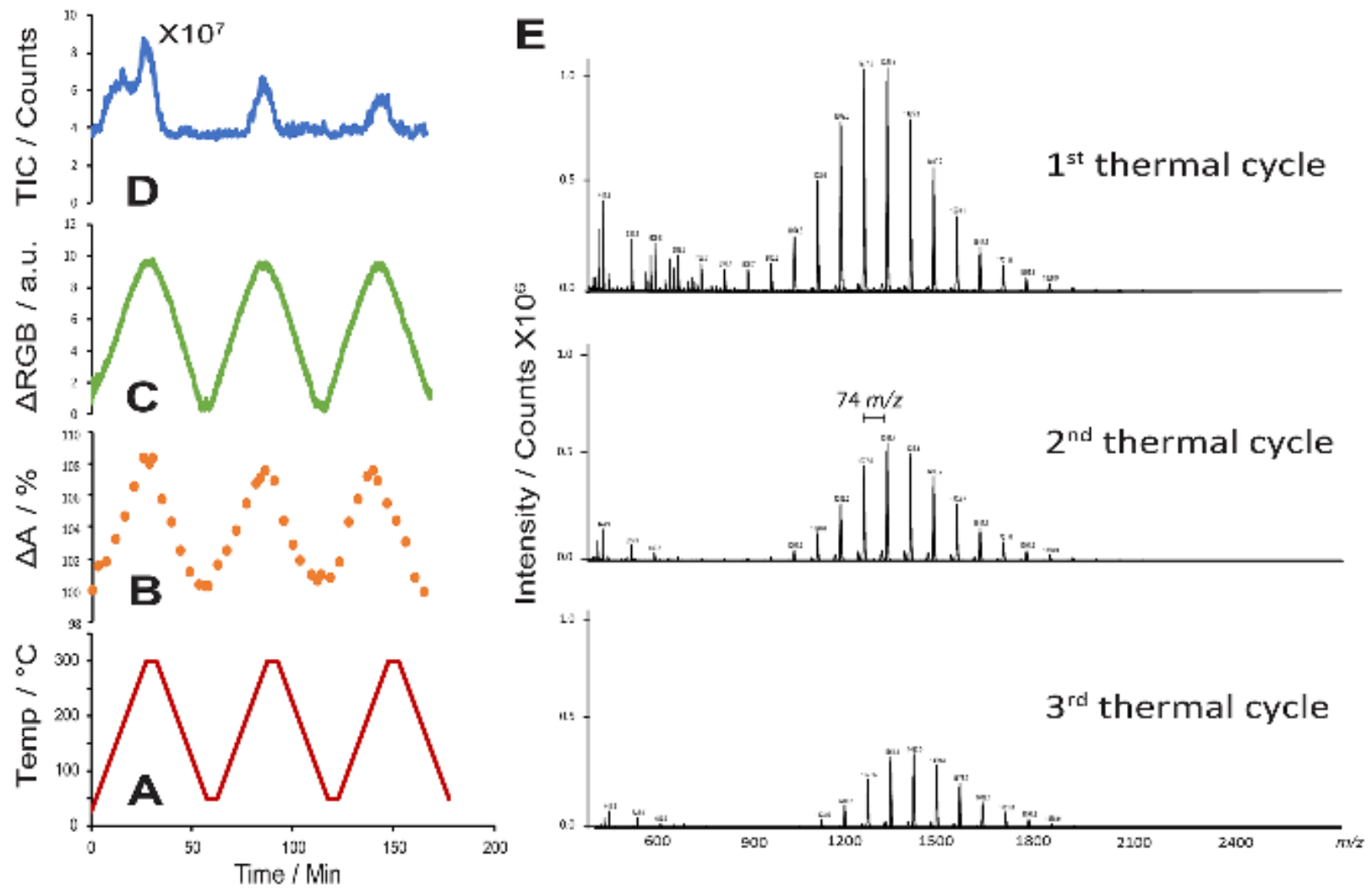

Figure 6 Thermal cycling of cookware grade silicone. (A - temperature, $B-$ area, $C-\triangle R G B$ and DTIC, E- average mass spectra)

This work demonstrates the unparalleled capability of the HDM system to combine physical and chemical information from a single experiment. This has allowed simultaneous measurement of the CTE in combination with chemical characterisation of evolved species. This would not be possible with either DART-MS or thermomechanical methods alone. 


\subsection{Microplastics}

Samples of sand were collected from two different beach locations on the east coast of England. The low-density components were isolated using a literature flotation method ${ }^{20}$ and the resultant material was then subjected to a linear heating programme of $20{ }^{\circ} \mathrm{Cmin}^{-1}$ between 30 and $550 \stackrel{\circ}{\circ}$.

Figure 7A shows four selected micrographs $(1 \times 1.5 \mathrm{~mm})$ recorded at intervals over the

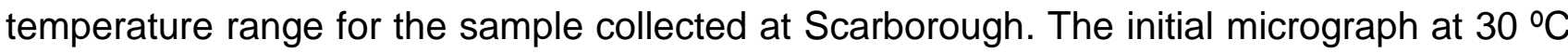
shows a varied range of flotation products (further images are shown in Fig. S6). As the temperature increases changes in morphology were observed in some of the particulates, indicating the presence of non-mineral species. At $240{ }^{\circ} \mathrm{C}$ melting is evident in some of the particulates and there are significant colour changes by $275 \stackrel{\circ}{\circ} \mathrm{C}$. After $400{ }^{\circ} \mathrm{C}$ these particulates are no longer visible.

Averaged mass spectra at different temperature points were examined for indicators of commonly-occurring polymers. Where these were observed, the data were interrogated for extracted ion chromatograms (EICs) of known oligomer $\mathrm{m} / \mathrm{z}$ values. In this sample, a series of ions consistent with polystyrene was observed (separated by $104 \mathrm{~m} / \mathrm{z}$, see Fig. 2C). Three selected EICs are shown in Fig. 7B (corresponding to $n=3,4$ and 5). It can be seen that a large increase in ion intensity occurs around the melting point of polystyrene $\left(240{ }^{\circ} \mathrm{C}\right) .{ }^{27}$ These ions are no longer present above $400^{\circ} \mathrm{C}$. This is consistent with visual changes seen in the micrographs. No other significant polymeric material was found in the sample. 


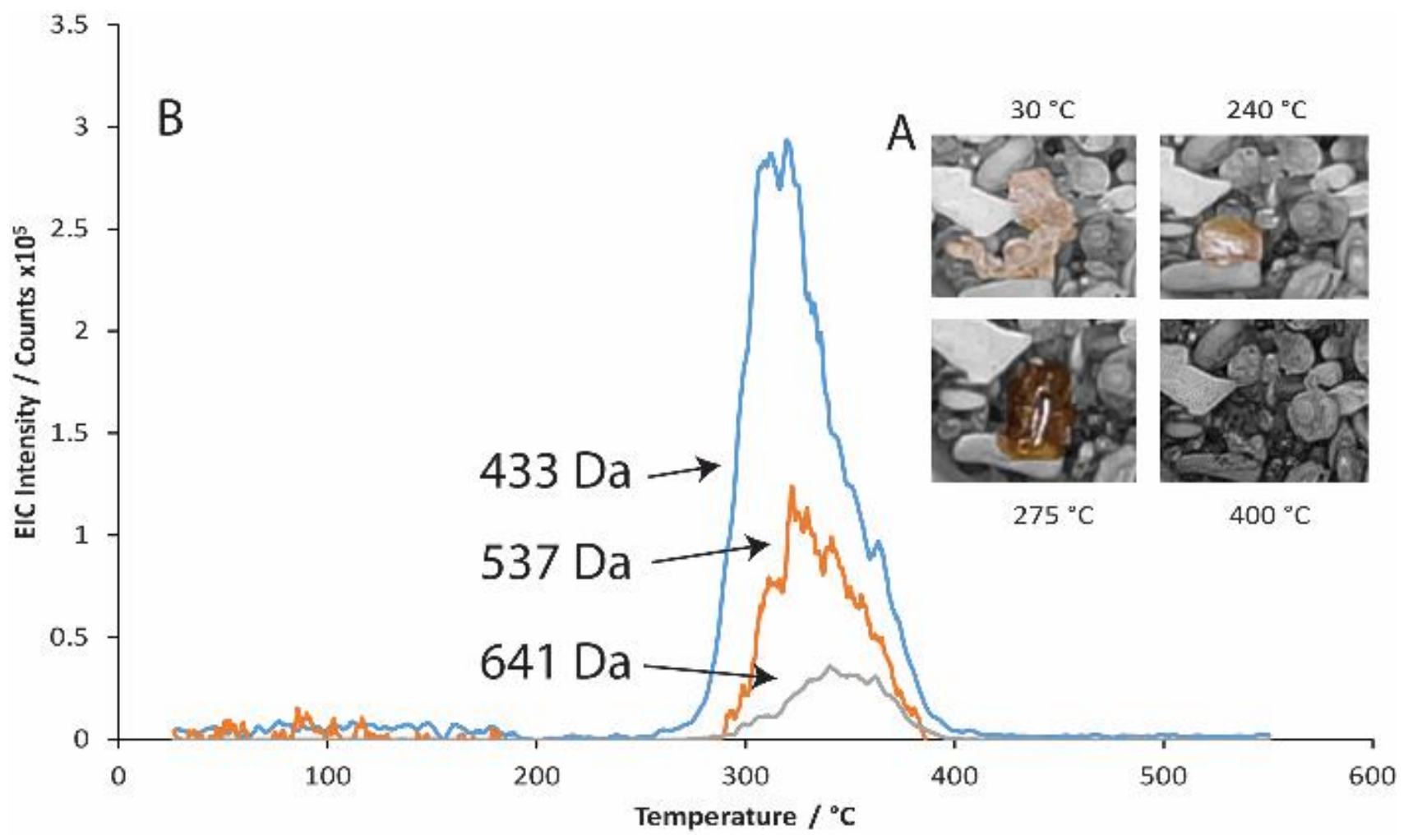

Figure 7 HDM analysis of flotation products extracted from beach 1. A) Greyscale micrographs showing polymeric species (highlighted in colour) melting; B) EIC profiles of selected polystyrene oligomers plotted as a function of temperature, $433 \mathrm{Da}\left[\mathrm{C}_{33} \mathrm{H}_{36}+\mathrm{H}\right]^{+}$(Blue), $537 \mathrm{Da}\left[\mathrm{C}_{41} \mathrm{H}_{44}+\mathrm{H}\right]^{+}$(Orange) and $641 \mathrm{Da}$ $\left[\mathrm{C}_{49} \mathrm{H}_{52}+\mathrm{H}\right]^{+}$(Grey) (micrographs in Fig. S6, average mass spectrum in Fig. S6b).

Figure 8 shows a comparable experiment using sand from Reighton. Selected micrographs are shown in Fig. 8A (see also Fig. S7). Again, non-mineral components were identified and analysis indicated the presence of polyethylene (Fig. 8B). 


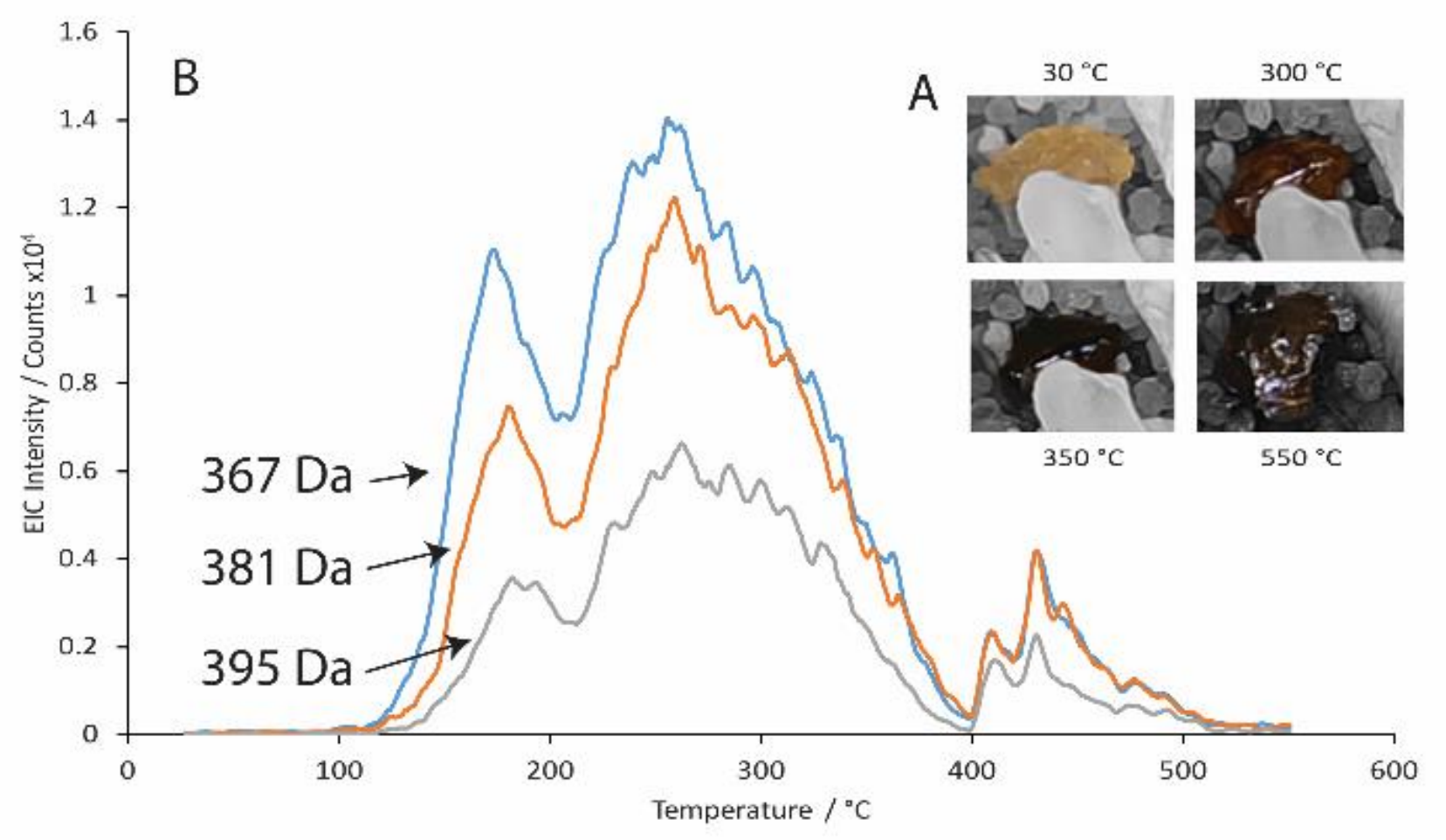

Figure 8 HDM analysis of flotation products extracted from beach 2. A) Greyscale micrographs showing polymeric species (highlighted in colour) melting and degrading; B) EIC profiles of representative polyethylene ions plotted as a function of temperature, $366 \mathrm{Da}\left[\mathrm{C}_{26} \mathrm{H}_{54}\right]^{-}$(Blue), $380 \mathrm{Da}\left[\mathrm{C}_{27} \mathrm{H}_{56}\right]^{-}$(Orange) and $394 \mathrm{Da}\left[\mathrm{C}_{28} \mathrm{H}_{58}\right]^{-}$(Grey) (micrographs in Fig. S7, average mass spectrum in Fig S7b)

Polyethylene oligomers (separated by $14 \mathrm{~m} / \mathrm{z}$, see Fig. 2B) are seen over a wide temperature range. A sharp increase in ion intensity is observed around $100^{\circ} \mathrm{C}$ which is consistent with the literature melting point of typical low-density PE used commercially. The ion intensity reaches a maximum at approximately $270 \stackrel{\circ}{\circ}$ before declining. A dip in the EIC profiles around $200{ }^{\circ} \mathrm{C}$ is attributed to a suppression effect; two peaks tentatively assigned as palmitic and

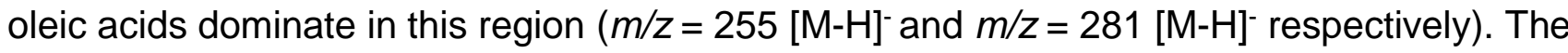
small final event after $400^{\circ} \mathrm{C}$ is likely to be linked to decomposition products sharing the same nominal masses as PE oligomers, visually consistent with the presence of a tar-like substance forming (see micrograph at $550{ }^{\circ} \mathrm{C}$ ). There was no evidence of other polymeric materials observed in the mass spectra.

These are preliminary results and are based on small sample sizes, but demonstrate the potential of HDM in this type of environmental analysis. 


\section{CONCLUSIONS}

HDM is a powerful new technique for obtaining combined mass spectral and optical data as a function of temperature. The potential for this system to be used for the analysis of polymers has been demonstrated using a variety of different samples. Physical and chemical changes in two silicone samples as a function of temperature were characterised from ambient temperature to $550^{\circ} \mathrm{C}$. A novel optical method of determining the coefficient of thermal expansion was developed and gave results consistent with both literature values and conventional measurement techniques. Thermal cycling experiments revealed that oligomer evolution declined with repeated cycling of the same sample. The method was extended to the study of microplastics from environmental samples collected from beaches on the east coast of the UK. Although this was a preliminary study, the HDM successfully identified polystyrene and polyethylene in the sand samples with less sample preparation required than with conventional methods of characterisation.

HDM has a range of beneficial features (including the lack of sample preparation required, flexibility in sample sizes which can be accommodated, precisely-controlled heating and cooling, sub-ambient capability for analysis of volatile species) which we intend to exploit in the analysis of a broader range of environmental samples (e.g. organic contaminants in soil).

\section{References}

1. Cody R, Laramée $H$, Durst $H$. Versatile new ion source for the analysis of materials in open air under ambient conditions. Analytical Chemistry. 2005; 77(8): 2297-2302.

2. Domin M, Cody R. Direct Analysis in Real-Time (DART $\left.{ }^{\circledR}\right)$. In: Ambient lonisation Mass Spectrometry. Vol Number 2; Cambridge, England; The Royal Society of Chemistry; 2014:2357.

3. Lennert E, Bridge CM. Rapid screening for smokeless powders using DART-HRMS and thermal desorption DART-HRMS. Forensic Chemistry. 2019; 13: 100148

4. Guo T, Yong W, Jin Y, et al. Applications of DART-MS for food quality and safety assurance in food supply chain. Mass Spectrometry Reviews. 2017; 36: 161-187.

5. Li L, Liu S, Ma L, Guo Y, Wang Y, Liu S. Application of direct analysis in real time-orbitrap mass spectrometry combined with multivariate data analysis for rapid quality assessment of Yuanhu Zhitong Tablet. International Journal of Mass Spectrometry. 2017; 421: 33-39.

6. Gross JH. Direct analysis in real time - a critical review on DART-MS. Analytical and Bioanalytical Chemistry. 2014; 406(1): 63-80. 
7. Harding LP, Parkes GMB, Townend JD. Parameters affecting ion intensities in transmission-mode direct analysis in real-time mass spectrometry. Analyst. 2014; 139: 41764180.

8. Ashton GP, Harding LP, Parkes GMB. An integrated hot-stage microscope-direct analysis in real-time mass spectrometry system for studying the thermal behaviour of materials. Analytical Chemistry. 2017; 89: 13466-13471

9. Charsley EL, Kamp ACF. A versatile hot stage microscope unit. In: Thermal analysis (Vol 1) Proceedings third ICTA Davos. Springer, Basel; 1972: 499-513.

10. Haines PJ. Simultaneous differential scanning calorimetry and reflected light intensity (DSC-RLI) in the study of inorganic materials. Thermochimica Acta. 1999: 340-341: 285-292. 11. Thermal Methods Research YouTube channel, https://www.youtube.com/channel/UCJ3DoKbw8NPadEzqYSELEGw?. Accessed April 12th 2019.

12. Haines PJ, Skinner GA. Simultaneous differential scanning calorimetry and reflected light intensity measurement. Thermochimica Acta. 1982; 59: 343-359.

13. Maric M, Marano J, Cody RB, Bridge C. DART-MS: A new analytical technique for forensic paint analysis. Analytical Chemistry. 2018; 90: 6877-6884.

14. Fazey FMC, Ryan PG. Biofouling on buoyant marine plastics: An experimental study into the effect of size on surface longevity. Environmental Pollution. 2016; 210: 354-360.

15. Eriksen M, Lebreton LCM, Carson HS, et al. Plastic pollution in the world's oceans: More than 5 trillion plastic pieces weighing over 250,000 tons afloat at sea. PLOS One. 2014; 0111913

16. Frias JPGL, Nash R. Microplastics: Finding a consensus on the definition. Marine Pollution Bulletin. 2019; 138: 145-147.

17. Carbery M, O'Connor W, Thavamani P. Trophic transfer of microplastics and mixed contaminants in the marine food web and implications for human health. Environmental International. 2018; 115: 400-409.

18. Fries E, Dekiff JH, Willmeyer J, Nuelle M-T, Ebert M, Remy D. Identification of polymer types and addatives in marine microplastic particles using pyrolysis-GC/MS and scanning electron microscopy. Environmental Science Processes \& Impacts. 2013; 15: 1949-1956.

19. Majewsky M, Bitter H, Eiche E, Horn H. Determination of microplastic polyethylene (PE) and polypropylene (PP) in environmental samples using thermal analysis (TGA-DSC). Science of the Total Environment. 2016; 568: 507-511. 
20. Coppock RL, Matthew C, Lindeque PK, Queiros AM, Galloway TM. A small-scale, portable method for extracting microplastics from marine sediments. Environmental Pollution. 2017; 230; 829-837

21. Camino G, Lomakin SM, Lazzari M. Polydimethylsiloxane thermal degradation Part 1. Kinetic aspects. Polymer. 2001; 42(6): 2395-2402.

22. Jakob A, Crawford EA, Gross JH. Detection of polydimethylsiloxanes transferred from silicone-coated parchment paper to baked goods using direct analysis in real time mass spectrometry. Journal of Mass Spectrometry. 2016; S1: 298-304

23. Tobushi H, Pieczyska E, Ejiri Y, Sakuragi T. Thermomechanical properties of shapememory alloys and polymers and their composites. Mechanics of Advanced Materials and Structures. 2009; 16(3): 236-247

24. Silex Silicones Ltd, personal communication

25. Lacerta UK, personal communication

26. Gross JH. Analysis of silicones released from household items and baby articles by direct analysis in real time-mass spectrometry. Journal of the American Society for Mass Spectrometry. 2015; 26:511-521

27. Asaletha R, Kumaran MG, Thomas S. Thermal behaviour of natural rubber/polystyrene blends: thermogravimetric and differential scanning calorimetric analysis. Polymer Degradation and Stability. 1198; 61: 431-439

\section{Acknowledgments}

The authors would like to thank the University of Huddersfield for funding (GPA). We would also like to thank Glynn van-de-Velde, Lacerta UK, for dynamic mechanical analysis measurements. 